\title{
Polymer-Assisted Fabrication of Silver Nanowire Cellular Monoliths: Towards Hydrophobic and Ultraflexible High-Performance Electromagnetic Interference Shielding Materials
}

Zhihui Zeng, ${ }^{\mathrm{a}, \mathrm{b}}$ Weiwei Li, ${ }^{\mathrm{c}} \mathrm{Na} \mathrm{Wu},{ }^{\mathrm{d}}$ Shanyu Zhao, ${ }^{\mathrm{b}}$ and Xuehong $\mathrm{Lu}^{\mathrm{a} *}$

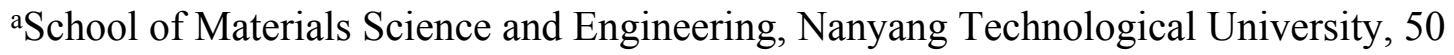

Nanyang Avenue, Singapore 639798, Singapore

bEmpa, Swiss Federal Laboratories for Materials Science and Technology

Überlandstrasse 129, 8600 Dübendorf, Switzerland

'IMPACT Lab, Computer, Electrical and Mathematical Sciences and Engineering

(CEMSE) Division, King Abdullah University of Science and Technology (KAUST),

Thuwal 23955-6900, Kingdom of Saudi Arabia.

${ }^{\mathrm{d} D e p a r t m e n t ~ o f ~ C h e m i s t r y, ~ S w i s s ~ F e d e r a l ~ I n s t i t u t e ~ o f ~ T e c h n o l o g y ~ i n ~ Z u r i c h ~(E T H ~}$

Zürich), 8092 Zürich, Switzerland

*Corresponding author. E-mail address: asxhlu@,ntu.edu.sg(Xuehong Lu). 


\begin{abstract}
Metal nanofibers with excellent electrical conductivity and superior mechanical flexibility have great potentials for fabrication of lightweight, flexible and high-performance electromagnetic interference (EMI) shielding architectures. The weak interactions and large contact resistance among the wires, however, hinder their assembly into robust and high-performance EMI shielding monoliths. In this work, we used low fractions of polymers to assist the construction of lightweight, flexible, and highly conductive silver nanowire $(\mathrm{AgNW})$ cellular monoliths with significantly enhanced mechanical strength and EMI shielding effectiveness (SE). The normalized surface specific SE of our AgNW-based cellular monoliths can reach up to $20522 \mathrm{~dB} \cdot \mathrm{cm}^{2} / \mathrm{g}$, outracing that of most shielding materials ever reported. Moreover, this robust conductive framework enabled the successful fabrication of hydrophobic, ultraflexible and highly stretchable aerogel/polymer composites with outstanding EMI SE even at an extremely low AgNW content. Thus, this work demonstrated a facile and efficient strategy for assembling metal nanofiber-based functional high-performance EMI shielding architectures.
\end{abstract}

Keywords: Electromagnetic interference, flexible, silver nanowires, polymer, porous 


\section{Introduction}

Electromagnetic waves generated by electronic appliances can cause electromagnetic interference (EMI) issues that may interrupt the normal work of electronic devices or even have some negative influences on human health. ${ }^{1-4}$ Lightweight high-performance EMI shielding materials, which possess high EMI shielding effectiveness (SE), excellent mechanical properties and some additional functionalities such as hydrophobicity and ultraflexibility, are hence highly demanded for electronic appliances, such as laptops, wireless telecommunication equipment, aircraft or spacecraft electronics, and smart and portable electronic devices. ${ }^{5-10}$ To achieve such advanced EMI shielding materials, various types of electric-conductive nanomaterials, such as carbon nanomaterials, ${ }^{11-27}$ transition-metal carbides, ${ }^{28-31}$ and metal nanomaterials ${ }^{32,33}$ have been combined with polymers to make conductive polymer composites (CPCs) with excellent mechanical flexibility and processability. In particular, the metal nanofibers ${ }^{33-38}$ exhibit favourable characteristics, such as ultrahigh aspect ratio, extraordinary electrical conductivity, and excellent mechanical flexibility, and thus, they have attracted great attention for constructing functional CPCs for EMI shielding applications. ${ }^{39-43}$

Among various approaches for the fabrication of CPCs, dispersing the metal nanofibers in polymer matrix to form sufficient percolation conductive networks is the main strategy. ${ }^{44-46}$ The resultant composites exhibit high EMI $\mathrm{SE}$ at the nanomaterial contents above a certain percolation threshold. ${ }^{11}$ In 
addition, micrometre-sized pores could also be introduced to generate substantial void-cell wall interfaces, which benefit the multiple reflection (multi-reflection) of the incident EM waves. ${ }^{8,20,33,44-49}$ For instance, typical porous CPCs, such as polyimide (PI)/silver nanowire $(\mathrm{AgNW})^{44}$, nanocellulose/ $\mathrm{AgNW}^{46}$, and polyurethane (PU)/AgNW ${ }^{45}$ composites, consist of conductive fillers dispersed in polymer matrix with micrometer-sized pores. The combination of the substantial charge carriers resulting from the metal nanowires, the interfacial dipoles derived from the polarization effect $17,23,50,51$ at the interface between the fillers and matrix, and the efficient multi-reflection leads to high EMI shielding performance of the porous CPCs. In particular, biomimetic anisotropic cellular monoliths such as microhoneycomb aerogels have been designed to boost the multi-reflections of EM waves in a particular direction of the architectures, ${ }^{21}, 23,45,46$ and the enhanced EMI shielding effectiveness achieved using this approach demonstrated the potential of the cellular structures for high-performance EMI shields. However, the large content of the insulating polymers matrix inevitably plagues the excellent conductivity of the metal wires in the CPCs and ultimately deteriorates the potential of the metal nanowires for high-performance EMI shielding. In addition, the agglomeration of the metal nanomaterials in the polymer matrix is also difficult to avoid, resulting in poor mechanical, electrical, and EMI shielding performance of the CPCs. Some metal nanofibers/conducting carbon hybrid aerogels ${ }^{41,42}$ and metal wrapped polymer nanofibers cellular monoliths ${ }^{33}$ 
have been fabricated to address these issues and showed promising results. Nevertheless, the reported methods involve complex instruments and/or harsh reaction conditions, such as high temperature or extreme $\mathrm{pH}$ environments and specific gas atmosphere. Thus, the rational design and efficient fabrication of metal nanofiber-based CPCs for high-performance EMI shielding application in a cost-effective way still remains a hot yet challenging topic in EMI shielding research field. In addition to EMI SE, integrated functionalities such as tuneable hydrophobicity, and ultraflexibility including bending, twisting, and stretching capabilities are highly desired for some emerging multifunctional systems with internet of things capabilities, such as soft robots, smart displays, and stretchable sensors.

In this work, we efficiently employed small amounts of polymers to assist in the construction of both ultralight and highly conductive AgNW-based aerogels with outstanding EMI shielding performance. Moreover, by tailoring their microstructures such AgNW-based aerogels could act as robust conductive frameworks for fabrication of lightweight, flexible, hydrophobic, and highly stretchable CPCs with excellent EMI shielding performance. Such ultralight high-performance EMI shielding materials and functional EMI shielding materials are practically very important for the EMI shielding field. Briefly, AgNW-based microhoneycomb aerogels are constructed first with the help of a very small fraction of polyvinyl pyrrolidone (PVP) using a facile unidirectional freeze-drying method ${ }^{52-54}$. The PVP-coated AgNWs are hierarchically 
three-dimensional (3D) interconnected to form lamellar cell walls, leading to superior mechanical flexibility, high electrical conductivity, and excellent EMI SE of the aerogels at fairly low densities. To further improve the interactions between the AgNWs, a small fraction of waterborne PU (WPU) binder is introduced, which results in increased nanoscale interfaces between the AgNWs and polymers and more lamellar cell walls with good structural integrity. As a result the WPU reinforced AgNW-based aerogels become more mechanically robust yet still fairly conductive. The aerogels exhibit a specific SE $(\mathrm{SSE})^{7,9-10}$, which is defined as SE divided by density of shielding materials, up to $4720 \mathrm{~dB} \cdot \mathrm{cm}^{3} / \mathrm{g}$ at a thickness of $2.3 \mathrm{~mm}$, and this value is much higher than that of most shielding materials at similar thicknesses. Finally, we demonstrate that the AgNW-based aerogels can be easily infiltrated with a stretchable polymer to form CPCs with excellent hydrophobicity, stability, and stretchability. The composites also demonstrate high EMI shielding performance at an ultralow AgNW loading, offering a promising route to meet the demands for emerging multifunctional electronics.

\section{Experimental}

2.1. Chemicals and Materials: PVP (average molecular weight of 55,000), hexadecyl trimethyl ammonium bromide (CTAB), ethylene glycol (EG, spectrophotometric grade, $\geqslant 99 \%)$ solution, and silver nitrate $\left(\mathrm{AgNO}_{3}, \mathrm{ACS}\right.$ reagent, $\geqslant 99 \%$ ) were bought from Sigma-Aldrich. WPU dispersion 
(Alberdingk U 3251), an aqueous, anionic, solvent-free, low viscosity dispersion of an aliphatic polyester-polyurethane without free isocyanate groups, was bought from Alberdingk Boley Inc., USA. Polydimethylsiloxane (PDMS, Sylgard 184 silicone elastomer) with the curing agent is bought from Dow corning.

2.2. Synthesis of PVP-coated AgNWs: The AgNWs were synthesized by a modified polyol procedure. Typically, PVP and CTAB were dissolved in an EG solution with concentrations of $0.2 \mathrm{M}$ and $3 \mathrm{mM}$, respectively. Then, $10 \mathrm{ml} \mathrm{EG}$ solution of $3.5 \mathrm{M} \mathrm{AgNO}_{3}$ was added at a speed of $0.2 \mathrm{~mL} / \mathrm{min}$ in the $400 \mathrm{~mL}$ as-prepared solution that had been heated to $160{ }^{\circ} \mathrm{C}$ via an oil bath. Constant temperature and gentle stirring were kept for 30 min followed by cooling the solution to room temperature. Finally, the solution was washed with acetone and distilled water for more than three times to remove the free PVP and ions in the dispersion to obtain an aqueous suspension of $\mathrm{AgNW}$ s coated with a thin layer of PVP.

2.3. Preparation of $\boldsymbol{P V P}-\boldsymbol{A g N W}$ aerogels. The AgNW aerogels with various shapes and densities were assembled by freezing the AgNW aqueous suspension casted in various moulds using liquid nitrogen, followed by freeze-drying at $-80{ }^{\circ} \mathrm{C}$ and $10 \mathrm{~Pa}$. When the suspension concentration was $0.75 \mathrm{wt} \%$, the density of the PVP-coated AgNW aerogels obtained from freeze-drying was $\sim 10 \mathrm{mg} / \mathrm{cm}^{3}$. 


\subsection{Preparation of WPU-reinforced AgNW aerogels (reinforced $\mathrm{AgNW}$}

aerogels). To prepare the reinforced AgNW aerogels, a desired amount of WPU aqueous dispersion was added to the AgNW suspension before freeze-drying. Typically, $0.75 \mathrm{wt} \% \mathrm{WPU}$ and $0.75 \mathrm{wt} \%$ PVP-coated AgNW suspensions were mixed and then, the mixture underwent the same freeze-drying process as mentioned above, leading to the formation of the reinforced AgNW aerogels at a similar density of $10 \mathrm{mg} / \mathrm{cm}^{3}$. Different ratios of the WPU to AgNWs could result in controllable WPU contents in the aerogels.

2.5. Preparation of reinforced AgNW aerogel/PDMS composites. The reinforced AgNW aerogels were infiltrated with PDMS to obtain the AgNW/PDMS composites. Briefly, pre-cured PDMS, a mixture of the base and the curing agent at a ratio of 10:1, was first degassed by a vacuum chamber in ice water bath reduce the effect of the curing on our composite preparation. It was then poured gently onto the reinforced AgNW aerogels and degassed for 20 min in vacuum oven, followed by the curing process at $70{ }^{\circ} \mathrm{C}$ for $4 \mathrm{~h}$. As a contrast, the AgNWs are also dispersed as fillers in the PDMS prepolymer by traditional mechanical stirring, followed by curing process to prepare the AgNW-dispersed rubber composites.

2.6. Characterization: Scanning electron microscopy (SEM) (JSM-7600F) and transmission electron microscopy (TEM, Tecnai G2 F20 U-TWIN) were used to characterize the microstructure. Thermal gravimetric analysis (TGA) curve 
was obtained through a thermogravimetric analyzer (TA instruments, USA) in nitrogen atmosphere. The resistance $(R)$ of the sample was measured by four-probe method with a Keithley 4200-SCS electrometer (Keithley, Cleveland, Ohio, USA) at room temperature to calculate the electrical conductivity. At least three samples were tested to get an average conductivity. Since the AgNWs were interconnected, the anisotropic porous architectures showed similar conductivity in various directions, and the infiltration of PDMS had negligible effect on the conductivity of the WPU-reinforced AgNW aerogels/PDMS composites. The compressive performance was characterized by a dynamic mechanical analyzer (DMA, TA Q800), and more than five samples were tested for each composite formulation. The compressive curves were obtained when the compressive direction is parallel to the aligned cell walls direction of the samples. When the aerogel/PDMS composites were subjected to uniaxial stretch by a home-built stretching stage, the resistance change was evaluated by measuring the resistance using the Keithley 4200-SCS. The composites with dimensions of $22.86 \mathrm{~mm} \times 10.16 \mathrm{~mm}$ (length $\times$ width) were employed to test EMI SE in the frequency range of $8.2-12.4 \mathrm{GHz}$ (X-band) using a waveguide method in a vector network analyser (Agilent E8363B PNA-L), and more than five samples for each component were tested. The samples were also bent by hand with more than 1000 cycles with a bending $\operatorname{radius}^{27}$ of around $10 \mathrm{~mm}$, and the resistance was tested every 50 bending cycles. EMI shielding performance was also obtained before and after the 
bending process.

\section{Results and discussion}

\subsection{Microstructure and Properties of PVP-AgNW Aerogels}

AgNWs with high aspect ratio of 700 are prepared as shown in Figure 1a-b. The high aspect ratio is beneficial for constructing of 3D porous architecture. In addition, a small fraction ( $\sim 4$ wt.\%) of PVP coated on the AgNWs (Figure 1c, d) leads to the stable dispersion of the AgNWs in water. The 3D decimetre-sized, flexible, low-density, and interconnected PVP-AgNW aerogels were directly assembled via unidirectional ice-templated freeze-drying method (Figure 1e-g, Figure S1). In the freeze-drying process, the unidirectional growth of ice crystals ${ }^{23}$ causes the assembly of submicron-sized AgNW lamellar cell walls, and aligned micrometre-sized pores. Herein, the aerogels show anisotropic microstructure in longitudinal plane (parallel to lamellar cell wall direction) (Figure 1e, Figure S2a-d), while the structure is fairly isotropic in transverse plane (perpendicular to lamellar cell wall direction), where the cell walls are randomly oriented (Figure 1f). In the lamellar cell walls, the nanowires intertwine and twist with each other. More AgNWs provide larger and more compact cell walls (Figure S2e-h) in the aerogels, while a higher water fraction leads to higher porosity and hence, lower density of the aerogels. The aerogels with considerable mechanical stability can be prepared with density as low as $4.0 \mathrm{mg} / \mathrm{cm}^{3}$, which corresponds 
to $0.038 \%$ of the weight of silver used, demonstrating more weight reduction than most metal-based porous materials ever reported ${ }^{35,36,55}$. It is observed that the amount of AgNWs in lamellar cell walls decreases with decreased aerogel density, and consequently, the thickness and integrity of the cell walls in the aerogels reduce (Figure S2a-h). For the aerogels with density higher than 20 $\mathrm{mg} / \mathrm{cm}^{3}$, the cell walls are dense, whereas loose and holey cell walls occur at density lower than $10 \mathrm{mg} / \mathrm{cm}^{3}$. In particular, the aerogels with density of $<4$ $\mathrm{mg} / \mathrm{cm}^{3}$ show obvious macroscopic shrinkage due to insufficient nanowires to construct cell walls (Figure S2i-1).

The microstructural characteristics of the aerogels lead to their excellent electrical conductivity and EMI shielding performance at low densities (Figure 1h). Higher conductivity of the AgNW aerogels can be obtained because of more intact and thicker cell walls as discussed before. The conductivity of the aerogels at a density of merely $4.0 \mathrm{mg} / \mathrm{cm}^{3}$ is around $90 \mathrm{~S} / \mathrm{m}$ and it can reach as high as $1400 \mathrm{~S} / \mathrm{m}$ at a density of $27.6 \mathrm{mg} / \mathrm{cm}^{3}$. Transverse EMI SE is obtained when the incident wave propagates along the transverse plane of the aerogels (perpendicular to the microhoneycomb pore channels) (Figure 1i), and it increases with increased density. The lightweight PVP-AgNW aerogels show an excellent EMI SE. Specifically, SE of the $2.3 \mathrm{~mm}$ thick aerogels at densities of $27.6,19.5$ and $10.0 \mathrm{mg} / \mathrm{cm}^{3}$ can reach $72.5,54.7$ and $36.4 \mathrm{~dB}$, respectively, far exceeding the typical requirement for commercial applications $(\sim 20 \mathrm{~dB})^{7,15}$. Generally, the total $\mathrm{SE}\left(\mathrm{SE}_{\mathrm{T}}\right)$ is influenced by reflection loss $\left(\mathrm{SE}_{\mathrm{R}}\right)$, absorption loss 
$\left(\mathrm{SE}_{\mathrm{A}}\right)$, and multi-reflection ability, which correspond to the mobile charge carriers, the electric (or magnetic) dipoles, and the reflections at various surfaces or interfaces, respectively ${ }^{39,46}$. Considering the anisotropic microstructure of the aerogels, longitudinal $\mathrm{SE}\left(\mathrm{L}-\mathrm{SE} E_{\mathrm{T}}, \mathrm{L}-\mathrm{SE}_{\mathrm{A}}\right.$ and $\mathrm{L}-\mathrm{SE}_{\mathrm{R}}$ ) values, corresponding to EMI SE when the incident electromagnetic waves propagate parallel to longitudinal plane (or microhoneycomb pore channels), are compared with the transverse $\mathrm{SE}\left(\mathrm{T}-\mathrm{SE} E_{\mathrm{T}}, \mathrm{T}-\mathrm{SE}_{\mathrm{A}}\right.$ and $\left.\mathrm{T}-\mathrm{SE} \mathrm{R}_{\mathrm{R}}\right)$ in Figure $1 \mathrm{j}$. Since incident electromagnetic waves propagating along transverse plane (perpendicular to the pore channels) can meet more lamellar cell walls, more multi-reflections of the waves occur and interact with the cell walls. Combining with the high electromagnetic wave absorption ability of the cell walls derived from the charge carriers of the AgNWs and interfacial polarization between the polymer and AgNWs, higher $\mathrm{T}-\mathrm{SE}_{\mathrm{A}}$ than $\mathrm{L}-\mathrm{SE}_{\mathrm{A}}$ is obtained. The $\mathrm{L}-\mathrm{SE}_{\mathrm{R}}$ and $\mathrm{T}-\mathrm{SE}_{\mathrm{R}}$ are similar at the same density because of the similar conductivities in both directions. This behaviour is similar to many other honeycomb-like porous architectures. ${ }^{23,} 52,54$ The aerogels can still keep high $\mathrm{SE}_{\mathrm{T}}$ values with significantly decreasing density, resulting in increased SSE values (Figure 1k). The SSE values can be larger than $2000 \mathrm{~dB} \cdot \mathrm{cm}^{3} / \mathrm{g}$ for $\mathrm{AgNW}$ aerogels, and the maximum transverse SSE can reach as high as $3638 \mathrm{~dB} \cdot \mathrm{cm}^{3} / \mathrm{g}$, which is one to two orders of magnitude higher than that of most shielding materials ever reported (Table S1).

\subsection{Microstructure and Properties of Reinforced AgNW aerogels}


Although, the PVP-AgNW aerogels show excellent flexibility and EMI shielding effectiveness, they suffer from low mechanical strength at low densities, which hinders their practical applications. More PVP can be added into the AgNW suspension, nevertheless, the brittleness of PVP at dry state can easily result in a fragile PVP-AgNW aerogel. Herein, a small fraction of WPU, which has excellent film formability and good mechanical strength and flexibility ${ }^{17,51}$, is introduced into the aerogels to further improve their mechanical properties. Compared with the aerogels without WPU (Figure 2a), the introduced WPU binders result in more lamellar cell walls with good integrity (Figure 2b, Figure S3). In addition to the excellent flexibility, the coactions of AgNWs and polymers can lead to significantly increased compressive strength and modulus of the porous architectures (Figure 2c, Figure S4a). The moduli of the reinforced AgNW aerogels containing 24.0 and $50.0 \mathrm{wt} \%$ WPU at the ultralow density of $10 \mathrm{mg} / \mathrm{cm}^{3}$ are 333 and $807 \mathrm{~Pa}$, respectively, which are $897 \%$ and $2316 \%$ higher respectively than that of the PVP-AgNW aerogels $(\sim 33.4 \mathrm{~Pa})$. Meanwhile, a small fraction of the WPU in the cell walls leads to only slightly decreased electrical conductivity of the AgNW-based porous architectures, which helps to maintain the high $\mathrm{SE}_{\mathrm{R}}$.

Other than benefiting mechanical properties, the lamellar cell walls with better integrity could also lead to more void-cell wall interfaces, promoting the multi-reflection of the incident electromagnetic waves in the transverse direction. In addition, AgNWs are well bound by the WPU binder in the cell walls 
of the reinforced AgNW aerogels, which introduces abundant interfaces between the AgNWs and polymers yet maintains the effective conductive AgNW networks. Therefore, by optimization of the microstructure of the aerogels, high interfacial polarization $^{17,37,50}$ and abundant charge carriers can be utilized simultaneously to boost wave absorption ability. Thus, the optimized microstructures can show great prospects for further improving EMI shielding performance of the aerogels. As shown in Figure 2d, when the WPU content is less than $50 \mathrm{wt} \%$, the T-SE $\mathrm{T}_{\mathrm{T}}$ increases initially with increased WPU content, while decreases much with further increased WPU content. The $\mathrm{SE}_{\mathrm{R}}$ decreases with increased WPU content, while dominant $\mathrm{SE}_{\mathrm{A}}$ shows an initial increase for the aerogels with a small fraction of WPU, resulting in the increased $\mathrm{SE}_{\mathrm{T}}$ (Figure 2e). However, too high a WPU content can result in more loss of charge carriers, leading to the decrease in $\mathrm{SE}_{\mathrm{A}}$ and $\mathrm{SE}_{\mathrm{T}}$. For the aerogels without $\mathrm{WPU}, \mathrm{SE}_{\mathrm{T}}$ and $\mathrm{SE}_{\mathrm{A}}$ are 36.4 and $26.5 \mathrm{~dB}$, respectively. $\mathrm{SE}_{\mathrm{T}}$ and $\mathrm{SE}_{\mathrm{A}}$ are 47.2 and $37.9 \mathrm{~dB}$, respectively, for the aerogels containing $24.0 \mathrm{wt} \% \mathrm{WPU}$, and 31.6 and $23.6 \mathrm{~dB}$, respectively, for the aerogels with $50.0 \mathrm{wt} \%$ WPU. In the wave propagation process (Figure S4b), the reflection happens before the absorption, e.g., around $90 \%$ of the total power is reflected first although the reflection capability $\left(\mathrm{SE}_{\mathrm{R}}\right)$ of aerogels is as low as $10 \mathrm{~dB} \cdot{ }^{17}$ Therefore, reflected power is higher than absorbed power even though the aerogels show higher absorption capability $\left(\mathrm{SE}_{\mathrm{A}}\right)$. The reflected power is slightly decreased while the absorbed power is slightly increased with increased WPU contents. However, the increase of absorbed power is more significant than the decrease of reflected power with the increase of WPU 
content up to $24 \mathrm{wt} \%$. Too high a WPU content will lead to more significant decrease of reflected power due to the decrease of conductivity. These lead to a minimum transmitted power $\left(\sim 10^{-5}\right)$ for the aerogels with $24.0 \mathrm{wt} \%$ WPU. The above results show that a small fraction of WPU in the AgNW aerogels can simultaneously enhance both their mechanical strength and EMI shielding performance.

Increased EMI SE of the reinforced AgNW aerogels contributes to significantly increased SSE (Figure S4c). The SSE of $2.3 \mathrm{~mm}$ thick aerogels can reach 3156 to $4724 \mathrm{~dB} \cdot \mathrm{cm}^{3} / \mathrm{g}$. Taking the effect of the thickness into consideration, EMI SE divided by the thickness $(\mathrm{SE} / d)$ are compared as a function of density for the typical shielding materials reported in literature (Figure 2f). At low densities, the WPU-reinforced AgNW aerogels show higher SE/d values than most shielding materials, and hence, their normalized surface SSE (SSE/d, defined as SE divided by the thickness and density) values are also much higher (Table S1). SSE/d of the WPU-reinforced AgNW aerogels can reach 20522 $\mathrm{dB} \cdot \mathrm{cm}^{2} / \mathrm{g}$, far higher than that of most shielding materials including some typical CPCs, commercial carbon aerogels, CNT sponge, CuNi alloy aerogels, and solid copper (Table S1). Considering the attractive features of the reinforced AgNW aerogels, including their ultrahigh EMI shielding performance (satisfactory SE and excellent SSE), decent mechanical strength, excellent flexibility, and the ease of preparation and controlling microstructures, they have great potentials for EMI shielding applications, 
where lightweight, flexible, and high shielding performance are required. Moreover, they can serve as robust conductive frameworks for preparation of flexible, stretchable, and durable high-performance EMI shielding CPCs as follows.

\subsection{Functionalities and EMI Shielding Properties of AgNW/PDMS}

\section{Composites}

With the significantly enhanced mechanical strength and structural integrity, the polymer-reinforced AgNW aerogels can be easily infiltrated with PDMS to form conductive rubber composites. The composite preparation consists two stages: (1) infiltration of the PDMS prepolymer into a piece of WPU-reinforced AgNW aerogel (containing $24 \mathrm{wt} \% \mathrm{WPU}$ ), and (2) subsequent curing of the prepolymer, as shown in Figure 3a. In both stages, WPU plays a vital role in preventing the AgNW scaffold from collapsing or suffering severe deformation. The high conductivity and desired microstructures of the AgNW aerogel can hence be maintained in the rubber composite, which are important for achieving high EMI shielding performance. By contrast, macroscopic deformation or microstructural damage of low-density AgNW aerogels are observed without WPU as the nanoglue due to the stresses incurred in the infiltration and curing of PDMS, deteriorating the properties of the corresponding rubber composites. In addition, the efficient infiltration of the PDMS could not only support the whole architectures, but also introduce excellent hydrophobicity (water contact 
angle of $\sim 110^{\circ}$ ). It has been well established that the efficient hydrophobicity is beneficial for the stability and reliability of the electronics in practical applications $^{56,57}$.

For many applications, flexible EMI shielding materials are desired to exhibit not only bendability and twistability but also stretchability. From the images in Figure 3b-d, we can see that our AgNW/PDMS composites exhibit excellent flexibility including bendability, twistability and high stretchability of more than $60 \%$. This implies the robust interactions between the WPU-reinforced AgNW framework and PDMS. Cross-sectional views of the microstructures of the rubber composite show that the WPU-reinforced AgNW framework is well embedded in the PDMS matrix, further manifesting the strong adhesion between the AgNW framework and PDMS (Figure 3e-g). The alignment of the AgNW-based cell walls can be well retained in the composite. The results clearly demonstrate that the WPU-reinforced AgNW aerogels can be well infiltrated with PDMS to produce bi-continuous CPCs with excellent mechanical strength (Figure S5) and flexibility, which can be attributed to the outstanding mechanical properties of the PDMS matrix, mechanical robustness of the WPU-reinforced AgNW framework, and the efficient interfaces between the two components.

In the process of composite preparation, the thickness and AgNW content of the composites can be easily controlled to tune the EMI SE (Figure 4a-b). The composites with larger thicknesses contain higher loadings of conductive 
AgNWs to interact with the incident electromagnetic waves, causing the increase of EMI SE. EMI SE of the $2 \mathrm{~mm}$ thick aerogel/PDMS composite can reach a value above $45 \mathrm{~dB}$, which is fairly close to the $\mathrm{SE}$ of the pristine aerogel without infiltrated PDMS. This further suggests that the microstructural characteristics of the aerogel are well retained in the aerogel/PDMS composites. The AgNW content of the CPCs can be estimated from the density of the WPU-reinforced AgNW aerogels, and it varies from 0.51 to $1.85 \mathrm{wt} \%$. Notably, the volume fractions of the AgNWs in the composites are only about 0.07 to $0.26 \mathrm{vol} \%$. The CPC with a higher AgNW content means that its AgNW framework has a higher density, which leads to higher $\mathrm{SE}_{\mathrm{A}}$ and $\mathrm{SE}_{\mathrm{R}}$ for the CPC, and hence higher $\mathrm{SE}_{\mathrm{T}}$. Remarkably, extremely low AgNW content of $0.51 \mathrm{wt} \%(0.07 \mathrm{vol} \%)$ in the flexible CPCs can still contribute to the high EMI SE due to the performed, interconnected AgNW-based conductive framework (Table S2). By contrast, the composites formed by dispersing AgNWs as fillers in the PDMS matrix do not show obvious electrical conductivity and EMI shielding function even when the AgNW content is $1 \mathrm{wt} . \%$. Compared with the total amount of conductive fillers in reported $\mathrm{CPCs}^{17}$, the EMI SE of the our rubber composite is higher even though the total amount of AgNWs is much lower (Table S2).

For flexible EMI shielding materials, their ability to maintain the original EMI shielding performance after repeated deformation cycles is essential for practical applications. Owing to the robust interactions between the 
AgNW-based framework and PDMS, our rubber composites exhibit marginal changes in electrical resistance ratio $\left(R / R_{0}\right.$, where $R$ and $R_{0}$ are the resistance after and before the mechanical deformation, respectively) and EMI SE even after 1000-cycle bending process (Figure 4c), demonstrating the excellent stability and advantages of our flexible composites for demanding EMI shielding applications. The electrical resistance of the AgNW /PDMS composites also show excellent reversibility and stability under the cyclic stretching/releasing deformations, and the change in resistance ratio is negligible even under high tensile strains of 30 to $60 \%$ (Figure $4 \mathrm{~d}$ ), which can be attributed to the interconnected $\mathrm{AgNW}$-based framework and their strong interactions with the PDMS matrix. In contrast, many filler-dispersed CPCs show an irreversible and large change of resistance when subjected to a large tensile strain ${ }^{58-60}$, which causes unstable EMI shielding performance. More importantly, the change in the resistance ratio of our rubber composite is still fairly small even after more than 1000 stretching/releasing cycles, resulting in a slight reduction in EMI SE (Figure 4e, f). Therefore, our AgNW/PDMS composites have great potentials for applications that need lightweight, highly flexible, stretchable, and stable materials with excellent EMI shielding performance.

\section{Conclusions}


We employed low fractions of polymers to assist in the construction of ultralight, flexible, and highly conductive AgNW-based microhoneycomb aerogels with outstanding EMI shielding performance. The aerogels enabled the successful preparation of multifunctional, high EMI shielding performance aerogel/PDMS composites. Herein, a small fraction of polymers can induce more intact lamellar cell walls and give rise to substantial interfacial area between AgNWs and polymers, whereas it reduces the electrical conductivity of the AgNW-based aerogels only slightly. This effectively improves the mechanical strength, EMI SE and SSE of the AgNW-based aerogels, which display remarkable EMI SE of $47.2 \mathrm{~dB}$ at merely a density of $10 \mathrm{mg} / \mathrm{cm}^{3}$ and a thickness of $2.3 \mathrm{~mm}$. The corresponding surface SSE is up to $20522 \mathrm{~dB} \cdot \mathrm{cm}^{2} / \mathrm{g}$, much higher than that reported for most shielding materials. Consequently, the AgNW aerogel/PDMS composites prepared from the robust conductive aerogel show outstanding EMI SE even at an extremely low AgNW content of 0.07 vol.\%. The strong interactions of the aerogel and PDMS also result in their excellent mechanical strength and stability, good hydrophobicity that benefits electronics reliability, and outstanding flexibility including bendability, twistability, and stretchability. This work offers a facile and efficient route for assembling metal nanofibers into multifunctional, high EMI shielding performance cellular monoliths, which has the great potential for application in next-generation smart electronics and devices. 


\section{ASSOCIATED CONTENT}

\section{(S) Supporting Information}

Preparation schematics, optical images, SEM images, mechanical performance, and EMI shielding performance of the as-manufactured AgNW-based aerogels and/or aerogel/PDMS composites; and the tables for EMI shielding performance of various shielding materials and various composite are included in the Supporting Information, which is available free of charge on the ACS Publications website at http://pubs.acs.org.

\section{AUTHOR INFORMATION}

\section{Corresponding Author}

E-mail: asxhlu@ntu.edu.sg (Xuehong Lu);

\section{ACKNOWLEDGMENTS}

Z.Z. and X.L. thank Nanyang Technological University, Singapore, for providing funding in the course of this work. Z.Z. and W.L. contributed equally to this work.

\section{REFERENCES}

1. Shahzad, F.; Alhabeb, M.; Hatter, C. B.; Anasori, B.; Man Hong, S.; Koo, C. M.; Gogotsi, Y. Electromagnetic Interference Shielding with 2D Transition Metal 
Carbides (MXenes). Science 2016, 353, 1137-1140.

2. Wen, B.; Cao, M.; Lu, M.; Cao, W.; Shi, H.; Liu, J.; Wang, X.; Jin, H.; Fang, X.;

Wang, W.; Yuan, J. Reduced Graphene Oxides: Light-weight and High-efficiency Electromagnetic Interference Shielding At Elevated Temperatures. Adv. Mater. 2014, 26, 3484-3489.

3. Namai, A.; Sakurai, S.; Nakajima, M.; Suemoto, T.; Matsumoto, K.; Goto, M.; Sasaki, S.; Ohkoshi, S.-i. Synthesis of An Electromagnetic Wave Absorber for High-Speed Wireless Communication. J. Am. Chem. Soc., 2009, 131, 1170-1173.

4. Zhang, Y.; Huang, Y.; Zhang, T.; Chang, H.; Xiao, P.; Chen, H.; Huang, Z.; Chen, Y. Broadband and Tunable High-performance Microwave Absorption Of An Ultralight and Highly Compressible Graphene Foam. Adv. Mater. 2015, 27, 2049-2053.

5. Ji, K.; Zhao, H.; Zhang, J.; Chen, J.; Dai, Z. Fabrication and Electromagnetic Interference Shielding Performance of Open-cell Foam of A Cu-Ni Alloy Integrated with CNTs. Appl. Surf. Sci. 2014, 311, 351-356.

6. Shen, B.; Zhai, W.; Tao, M.; Ling, J.; Zheng, W. Lightweight, Multifunctional Polyetherimide/Graphene@Fe3O4 Composite Foams for Shielding of Electromagnetic Pollution. ACS Appl. Mater. Interfaces 2013, 5, 11383-11391.

7. Chen, Z.; Xu, C.; Ma, C.; Ren, W.; Cheng, H. M. Lightweight and Flexible Graphene Foam Composites for High-performance Electromagnetic Interference Shielding. Adv Mater 2013, 25, 1296-1300.

8. Zhang, H. B.; Yan, Q.; Zheng, W. G.; He, Z.; Yu, Z. Z. Tough Graphene-polymer 
Microcellular Foams for Electromagnetic Interference Shielding. ACS Appl. Mater. Interfaces 2011, 3, 918-24.

9. Yang, Y.; Gupta, M. C.; Dudley, K. L.; Lawrence, R. W. Novel Carbon Nanotube-Polystyrene Foam Composites for Electromagnetic Interference Shielding. Nano Lett. 2005, 5, 2131-2134.

10. Yang, Y.; Gupta, M. C.; Dudley, K. L.; Lawrence, R. W. Conductive Carbon Nanofiber-Polymer Foam Structures. Adv. Mater. 2005, 17, 1999-2003.

11. Thomassin, J.-M.; Jérôme, C.; Pardoen, T.; Bailly, C.; Huynen, I.; Detrembleur, C. Polymer/Carbon based Composites as Electromagnetic Interference (EMI) Shielding Materials. Mater. Sci. Engineer. R Rep. 2013, 74, 211-232.

12. Chen, Y.; Zhang, H.-B.; Yang, Y.; Wang, M.; Cao, A.; Yu, Z.-Z. High-Performance Epoxy Nanocomposites Reinforced with Three-Dimensional Carbon Nanotube Sponge for Electromagnetic Interference Shielding. Adv. Funct. Mater. 2016, 26, 447-455.

13. Li, H.; Yuan, D.; Li, P.; He, C. High Conductive and Mechanical Robust Carbon Nanotubes/Waterborne Polyurethane Composite Films for Efficient Electromagnetic Interference Shielding, Comp. Part A Appl. Sci. Manuf. 2019, 121, 411-417.

14. Chung, D. D. L., Electromagnetic Interference Shielding Effectiveness of Carbon Materials. Carbon 2001, 39, 279-285.

15. Al-Saleh, M. H.; Saadeh, W. H.; Sundararaj, U. EMI Shielding Effectiveness of Carbon based Nanostructured Polymeric Materials: A Comparative Study. Carbon 2013, 60, 146-156. 
16. Wen, B.; Wang, X. X.; Cao, W. Q.; Shi, H. L.; Lu, M. M.; Wang, G.; Jin, H. B.; Wang, W. Z.; Yuan, J.; Cao, M. S. Reduced Graphene Oxides: the Thinnest and Most Lightweight Materials with Highly Efficient Microwave Attenuation Performances of the Carbon World. Nanoscale 2014, 6, 5754-5761.

17. Zeng, Z.; Chen, M.; Jin, H.; Li, W.; Xue, X.; Zhou, L.; Pei, Y.; Zhang, H.; Zhang, Z. Thin and Flexible Multi-walled Carbon Nanotube/Waterborne Polyurethane Composites with High-performance Electromagnetic Interference Shielding. Carbon 2016, 96, 768-777.

18. Liu, Z.; Bai, G.; Huang, Y.; Ma, Y.; Du, F.; Li, F.; Guo, T.; Chen, Y. Reflection and Absorption Contributions to the Electromagnetic Interference Shielding of Single-Walled Carbon Nanotube/Polyurethane Composites. Carbon 2007, 45, $821-827$.

19. Huang, Y.; Li, N.; Ma, Y.; Du, F.; Li, F.; He, X.; Lin, X.; Gao, H.; Chen, Y. The Influence of Single-walled Carbon Nanotube Structure on The Electromagnetic Interference Shielding Efficiency of Its Epoxy Composites. Carbon 2007, 45, $1614-1621$.

20. Ling, J.; Zhai, W.; Feng, W.; Shen, B.; Zhang, J.; Zheng, W. Facile Preparation of Lightweight Microcellular Polyetherimide/Graphene Composite Foams for Electromagnetic Interference Shielding. ACS Appl. Mater. Interfaces 2013, 5, $2677-2684$.

21. Li, X. H.; Li, X.; Liao, K. N.; Min, P.; Liu, T.; Dasari, A.; Yu, Z. Z. Thermally Annealed Anisotropic Graphene Aerogels and Their Electrically Conductive Epoxy 
Composites with Excellent Electromagnetic Interference Shielding Efficiencies. ACS Appl. Mater. Interfaces 2016, 8, 33230-33239.

22. Zeng, Z.; Wang, C.; Wu, T.; Han, D.; Lukovic, M.; Pan, F.; Siqueira, G.; Nyström G. Nanocellulose Assisted Preparation of Ambient Dried, Large-Scale and Mechanically Robust Carbon Nanotube Foams for Electromagnetic Interference Shielding. J. Mater. Chem. A, 2020, doi.10.1039/D0TA05961G.

23. Zeng, Z.; Jin, H.; Chen, M.; Li, W.; Zhou, L.; Zhang, Z. Lightweight and Anisotropic Porous MWCNT/WPU Composites for Ultrahigh Performance Electromagnetic Interference Shielding. Adv. Funct. Mater. 2016, 26, 303-310.

24. Hong, X.; Chung, D. D. L. Carbon Nanofiber Mats for Electromagnetic Interference Shielding. Carbon 2017, 111, 529-537.

25. Song, Q.; Ye, F.; Yin, X.; Li, W.; Li, H.; Liu, Y.; Li, K.; Xie, K.; Li, X.; Fu, Q.; Cheng, L.; Zhang, L.; Wei, B. Carbon Nanotube-Multilayered Graphene Edge Plane Core-Shell Hybrid Foams for Ultrahigh-Performance Electromagnetic-Interference Shielding. Adv. Mater. 2017, 29, 1701583.

26. Wu, Y.; Wang, Z.; Liu, X.; Shen, X.; Zheng, Q.; Xue, Q.; Kim, J. K. Ultralight Graphene Foam/Conductive Polymer Composites for Exceptional Electromagnetic Interference Shielding. ACS Appl. Mater. Interfaces 2017, 9, 9059-9069.

27. Zhou, B.; Han, X.; Li, L.; Feng, Y.; Fang, T.; Zheng, G.; Wang, B.; Dai, K.; Liu, C.; Shen, C., Ultrathin, Flexible Transparent Joule Heater with Fast Response Time Based on Single-Walled Carbon Nanotubes/Poly(Vinyl Alcohol) Film. Comp. Sci. Technol. 2019, 183, 107796 
28. Liang, L.; Han, G.; Li, Y.; Zhao, B.; Zhou, B.; Feng, Y.; Ma, J.; Wang, Y.; Zhang, R.; Liu, C., Promising Ti3C2Tx MXene/Ni Chain Hybrid with Excellent Electromagnetic Wave Absorption and Shielding Capacity. ACS Appl. Mater. Interfaces 2019, 11, 25399-25409.

29. Liang, L.; Yang, R.; Han, G.; Feng, Y.; Zhao, B.; Zhang, R.; Wang, Y.; Liu, C., Enhanced Electromagnetic Wave-Absorbing Performance of Magnetic Nanoparticles-Anchored 2D Ti3C2Tx MXene. ACS Appl. Mater. Interfaces 2020, 12 , 2644-2654.

30. Zhou, B.; Zhang, Z.; Li, Y.; Han, G.; Feng, Y.; Wang, B.; Zhang, D.; Ma, J.; Liu, C., Flexible, Robust, and Multifunctional Electromagnetic Interference Shielding Film with Alternating Cellulose Nanofiber and MXene Layers. ACS Appl. Mater. Interfaces 2020, 12, 4895-4905.

31. Zeng, Z.; Wang, C.; Siqueira, G.; Han, D.; Huch, A.; Abdolhosseinzadeh, S.; Heier, J.; Nüesch, F.; Zhang, C.; Nyström, G. Nanocellulose-MXene Biomimetic Aerogels with Orientation-Tunable Electromagnetic Interference Shielding Performance. Adv. Sci. 2020, 2000979.

32. Gao, J.; Luo, J.; Wang, L.; Huang, X.; Wang, H.; Song, X.; Hu, M.; Tang L.; Xue, H. Flexible, Superhydrophobic and Highly Conductive Composite Based on Non-Woven Polypropylene Fabric for Electromagnetic Interference Shielding. Chem. Eng. J., 2019, 364, 493-502.

33. Cho, S.; Kang, S.; Pandya, A.; Shanker, R.; Khan, Z.; Lee, Y.; Park, J.; Craig, S. L.; Ko, H. Large-Area Cross-Aligned Silver Nanowire Electrodes for Flexible, 
Transparent, and Force-Sensitive Mechanochromic Touch Screens. ACS Nano 2017, $11(4), 4346-4357$.

34. Choi, S.; Park, J.; Hyun, W.; Kim, J.; Kim, J.; Lee, Y. B.; Song, C.; Hwang, H. J.; Kim, J. H.; Hyeon, T.; Kim, D.-H. Stretchable Heater Using Ligand-Exchanged Silver Nanowire Nanocomposite for Wearable Articular Thermotherapy. ACS Nano 2015, 9 (6), 6626-6633.

35. Tang, Y.; Gong, S.; Chen, Y.; Yap, L. W.; Cheng, W. Manufacturable Conducting Rubber Ambers and Stretchable Conductors from Copper Nanowire Aerogel Monoliths. ACS Nano 2014, 8, 5707-5714.

36. Tang, Y.; Yeo, K. L.; Chen, Y.; Yap, L. W.; Xiong, W.; Cheng, W., Ultralow-Density Copper Nanowire Aerogel Monoliths with Tunable Mechanical and Electrical Properties. J. Mater. Chem. A 2013, 1, 6723.

37. Mutiso, R. M.; Sherrott, M. C.; Rathmell, A. R.; Wiley, B. J.; Winey, K. I. Integrating Simulations and Experiments To Predict Sheet Resistance and Optical Transmittance in Nanowire Films for Transparent Conductors. ACS Nano 2013, 7 (9), 7654-7663.

38. Jiu, J.; Sugahara, T.; Nogi, M.; Araki, T.; Suganuma, K.; Uchida, H.; Shinozaki, K. High-Intensity Pulse Light Sintering of Silver Nanowire Transparent Films on Polymer Substrates: The Effect of The Thermal Properties of Substrates on The Performance of Silver Films. Nanoscale 2013, 5 (23), 11820-11828.

39. Zeng, Z.; Jiang, F.; Yue, Y.; Han, D.; Lin, L.; Zhao, S.; Zhao, Y. B.; Pan, Z.; Li, C.; Nystrom, G.; Wang, J. Flexible and Ultrathin Waterproof Cellular 
Membranes Based on High-Conjunction Metal-Wrapped Polymer Nanofibers for Electromagnetic Interference Shielding, Adv. Mater. 2020, 32, 1908496.

40. Zhang, Y.; Tian, W.; Liu, L.; Cheng, W.; Wang, W.; Liew, K.M.; Wang, B.; Hu, Y.; Eco-Friendly Flame Retardant and Electromagnetic Interference Shielding Cotton Fabrics with Multi-Layered Coatings, Chem. Eng. J. 2019, 372, 1077-1090.

41. Wan, Y.-J.; Zhu, P.-L.; Yu, S.-H.; Sun, R.; Wong, C.-P.; Liao, W.-H.; Anticorrosive, Ultralight, and Flexible Carbon-Wrapped Metallic Nanowire Hybrid Sponges for Highly Efficient Electromagnetic Interference Shielding, Small 2018, 14, 1800534.

42. Wu, S.; Zou, M.; Li, Z.; Chen, D.; Zhang, H.; Yuan, Y.; Pei, Y.; Cao, A. Robust and Stable $\mathrm{Cu}$ Nanowire@Graphene Core-Shell Aerogels for Ultraeffective Electromagnetic Interference Shielding. Small 2018, 14, 1800634.

43. Lee, T. W.; Lee, S. E.; Jeong, Y. G. Highly Effective Electromagnetic Interference Shielding Materials Based on Silver Nanowire/Cellulose Papers. ACS Appl. Mater. Interfaces 2016, 8, 13123-13132.

44. Ma, J.; Zhan, M.; Wang, K. Ultralightweight Silver Nanowires Hybrid Polyimide Composite Foams For High-performance Electromagnetic Interference Shielding. ACS Appl. Mater. Interfaces 2015, 7, 563-576.

45. Zeng, Z.; Chen, M.; Pei, Y.; Seyed Shahabadi, S. I.; Che, B.; Wang, P.; Lu, X. Ultra-light and Flexible Polyurethane/Silver Nanowire Nanocomposites with Unidirectional Pores for Highly Effective Electromagnetic Shielding. ACS Appl. Mater. Interfaces 2017, 9, 32211-32219. 
46. Zeng, Z.; Wu, T.; Han, D.; Ren, Q.; Siqueira, G.; Nystrom, G. Ultralight, Flexible, and Biomimetic Nanocellulose/Silver Nanowire Aerogels for Electromagnetic Interference Shielding, ACS Nano 2020, 14, 2927-2938.

47. Zhang, L.; Liu, M.; Roy, S.; Chu, E. K.; See, K. Y.; Hu, X. Phthalonitrile-based Carbon Foam with High Specific Mechanical Strength and Superior Electromagnetic Interference Shielding Performance. ACS Appl. Mater. Interfaces 2016, 8, 7422-30.

48. Yan, D.-X.; Ren, P.-G.; Pang, H.; Fu, Q.; Yang, M.-B.; Li, Z.-M. Efficient Electromagnetic Interference Shielding of Lightweight Graphene/Polystyrene Composite. J. Mater. Chem. 2012, 22, 18772-18774.

49. Ameli, A.; Jung, P. U.; Park, C. B. Electrical Properties and Electromagnetic Interference Shielding Effectiveness of Polypropylene/Carbon Fiber Composite Foams. Carbon 2013, 60, 379-391.

50. Yousefi, N.; Sun, X.; Lin, X.; Shen, X.; Jia, J.; Zhang, B.; Tang, B.; Chan, M.;

Kim, J. K. Highly Aligned Graphene/Polymer Nanocomposites with Excellent Dielectric Properties for High-performance Electromagnetic Interference Shielding. Adv. Mater. 2014, 26, 5480-5487.

51. Hsiao, S.-T.; Ma, C.-C. M.; Tien, H.-W.; Liao, W.-H.; Wang, Y.-S.; Li, S.-M.; Huang, Y.-C. Using A Non-covalent Modification to Prepare A High Electromagnetic Interference Shielding Performance Graphene Nanosheet/Water-borne Polyurethane Composite. Carbon 2013, 60, 57-66.

52. Zhang, L. Q.; Yang, S. G.; Li, L.; Yang, B.; Huang, H. D.; Yan, D. X.; Zhong, G. J.; Xu, L.; Li, Z. M., Ultralight Cellulose Porous Composites with Manipulated 
Porous Structure and Carbon Nanotube Distribution for Promising Electromagnetic Interference Shielding. ACS Appl.Mater. Interfaces 2018, 10, 40156-40167.

53. Zhang, H.; Cooper, A. I. Aligned Porous Structures by Directional Freezing. Adv. Mater. 2007, 19, 1529-1533.

54. Zeng, Z.; Jin, H.; Chen, M.; Li, W.; Zhou, L.; Xue, X.; Zhang, Z., Microstructure Design of Lightweight, Flexible, and High Electromagnetic Shielding Porous Multiwalled Carbon Nanotube/Polymer Composites. Small 2017, 13, 201701388.

55. Gao, H. L.; Xu, L.; Long, F.; Pan, Z.; Du, Y. X.; Lu, Y.; Ge, J.; Yu, S. H. Macroscopic Free-standing Hierarchical 3D Architectures Assembled From Silver Nanowires by Ice Templating. Angew. Chem. Int. Ed. 2014, 53, 4561-4566.

56. Zhou, X.; He, C. Tailoring The Surface Chemistry and Morphology of Glass Fiber Membranes for Robust Oil/Water Separation Using Poly(Dimethylsiloxanes) as Hydrophobic Molecular Binders. J. Mater. Chem. A, 2018, 6, 607-615.

57. Liu, J.; Zhang, H.B.; Sun, R.; Liu, L.; Liu, Z.; Zhou, A.; Yu, Z.Z. Hydrophobic, Flexible, and Lightweight MXene Foams for High-Performance Electromagnetic-Interference Shielding. Adv. Mater. 2017, 29, 1702367.

58. Chen, M.; Zhang, L.; Duan, S.; Jing, S.; Jiang, H.; Luo, M.; and Li, C. Highly Conductive and Flexible Polymer Composites with Improved Mechanical and Electromagnetic Interference Shielding Performances. Nanoscale, 2014, 6, 3796-3803.

59. Li, P.; Du, D.; Guo, L.; Guo, Y.; and Ouyang, J. Stretchable and Conductive Polymer Films For High-Performance Electromagnetic Interference Shielding. $J$. 
Mater. Chem. C, 2016, 4, 6525-6532.

60. Zeng, Z.; Liu, M.; Xu, H.; Liao, Y.Z.; Duan, F.; Zhou, L.; Jin, H.; Zhang, Z.; Su, Z. Ultra-Broadband Frequency Responsive Sensor Based on Lightweight and Flexible Carbon Nanostructured Polymeric Nanocomposites. Carbon, 2017, 121, $490-501$ 

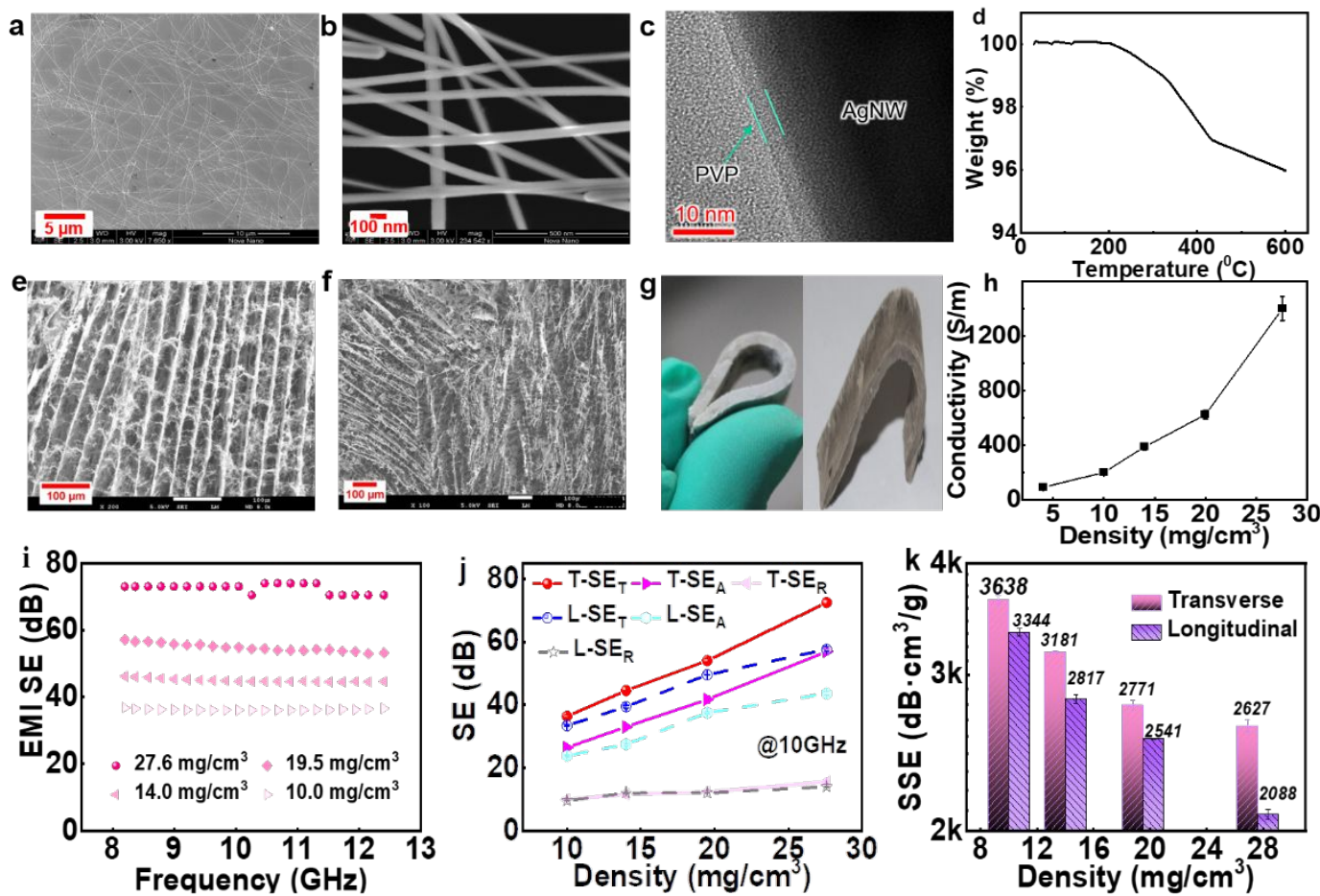

Figure 1. (a, b) SEM (scale bars are $5 \mu \mathrm{m}$ and $100 \mathrm{~nm}$, respectively) and (c) TEM images (scale bar is $10 \mathrm{~nm}$ ), and (d) TGA curve of the as-prepared AgNWs (diameter $\approx 50 \pm 12 \mathrm{~nm}$ and length $\approx 35 \pm 12 \mu \mathrm{m}$ ) with $\sim 4 \mathrm{wt} \%$ PVP. Microstructures of the PVP-AgNW aerogels in (e) longitudinal and (f) transverse planes. (Scale bars are $100 \mu \mathrm{m})$. (g) Optical images of the aerogels with density of $27.6 \mathrm{mg} / \mathrm{cm}^{3}$ (left) and $6.0 \mathrm{mg} / \mathrm{cm}^{3}$ (right); (h) electrical conductivity of the aerogels at various densities; (i) transverse EMI SE in the X-band, (j) transverse and longitudinal EMI SE at $10 \mathrm{GHz}$ frequency, and (k) transverse and longitudinal SSE of the aerogels with various densities at 10 GHz. 
a
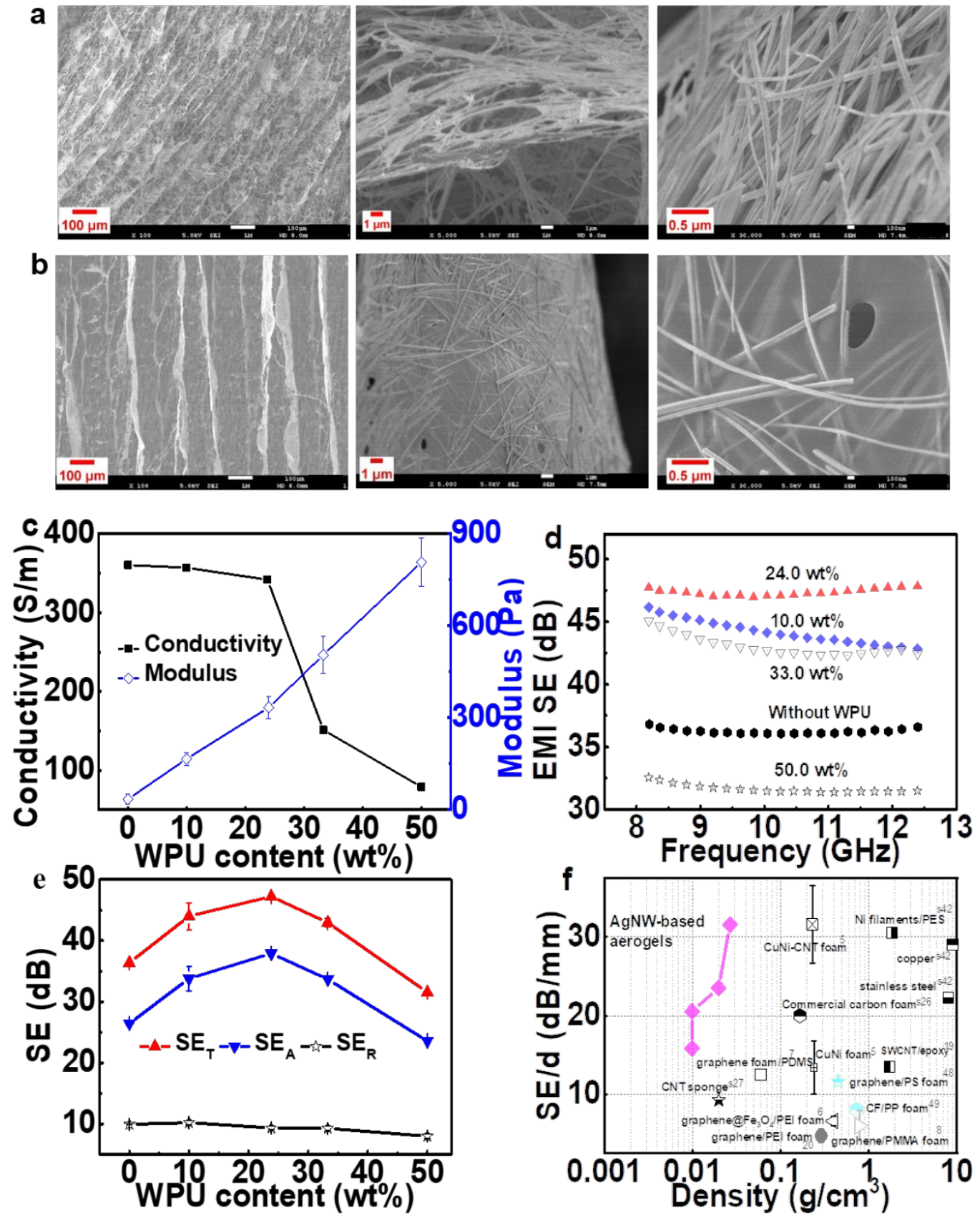

Figure 2. Microstructure of the AgNW-based aerogels at the density of 10 $\mathrm{mg} / \mathrm{cm}^{3}$ : (a) without WPU, (b) with $24.0 \mathrm{wt} \%$ WPU. (Scale bars are 100, 10, and $0.5 \mu \mathrm{m}$ from the left to right column, respectively.) Properties of the reinforced AgNW aerogels with various WPU contents at a density of 10 $\mathrm{mg} / \mathrm{cm}^{3}$ : (c) electrical conductivity and compressive modulus, (d) EMI shielding performance, and (e) $\mathrm{SE}_{\mathrm{T}}, \mathrm{SE}_{\mathrm{R}}$, and $\mathrm{SE}_{\mathrm{A}}$ at $10 \mathrm{GHz}$ frequency. (f) 
$\mathrm{SE} / d$ values of the reinforced AgNW aerogels and reported typical shielding materials with various densities.
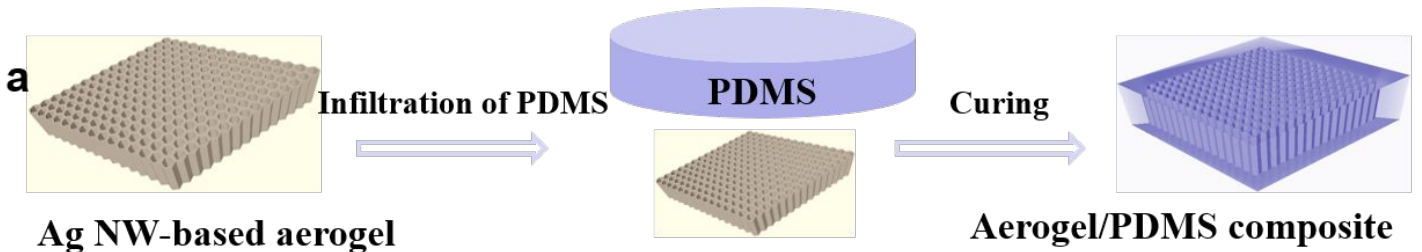

Ag NW-based aerogel
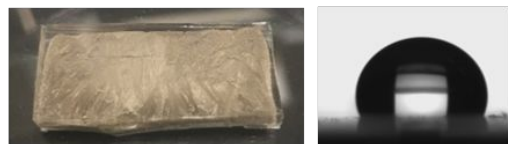

b

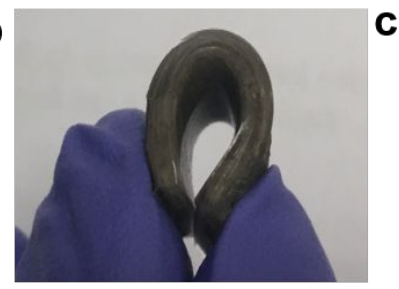

e

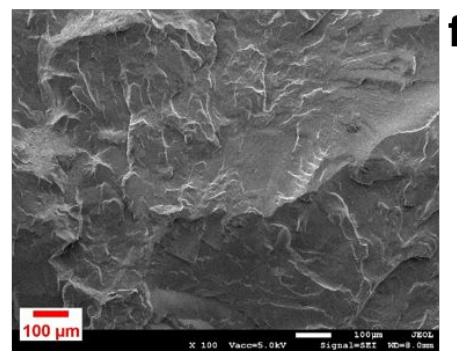

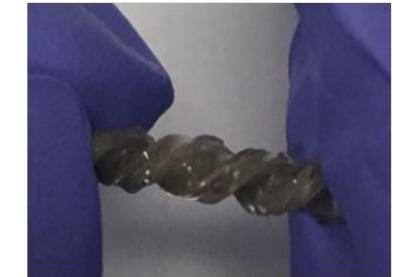

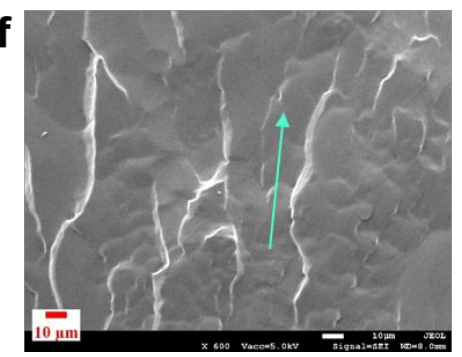

d

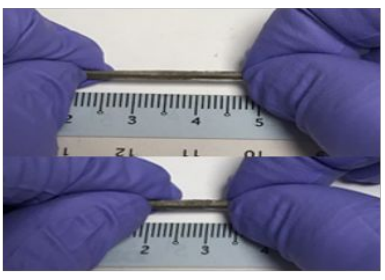

g

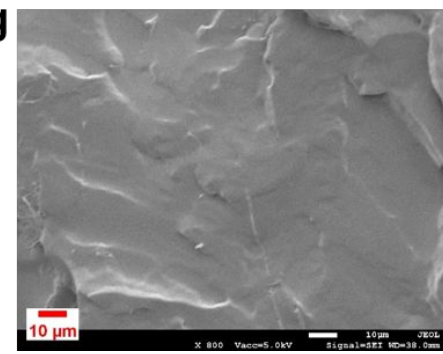

Figure 3. (a) Schematics showing the fabrication process of the reinforced AgNW aerogel/PDMS composites with water contact angle of $\sim 110^{\circ}$, and optical images of the composites showing (b) bendability, (c) twistability, and (d) stretchability (stretched to more than $60 \%$ strain). (e-g) SEM images for the cross-sectional cryogenically-fractured surface of the composites (arrow in image (f) indicates the direction of the aligned cell walls of the microhoneycomb aerogel in the composite). (Scale bars are 100, 10, and 10 $\mu \mathrm{m}$ for images e, $\mathrm{f}$, and $\mathrm{g}$, respectively.) 

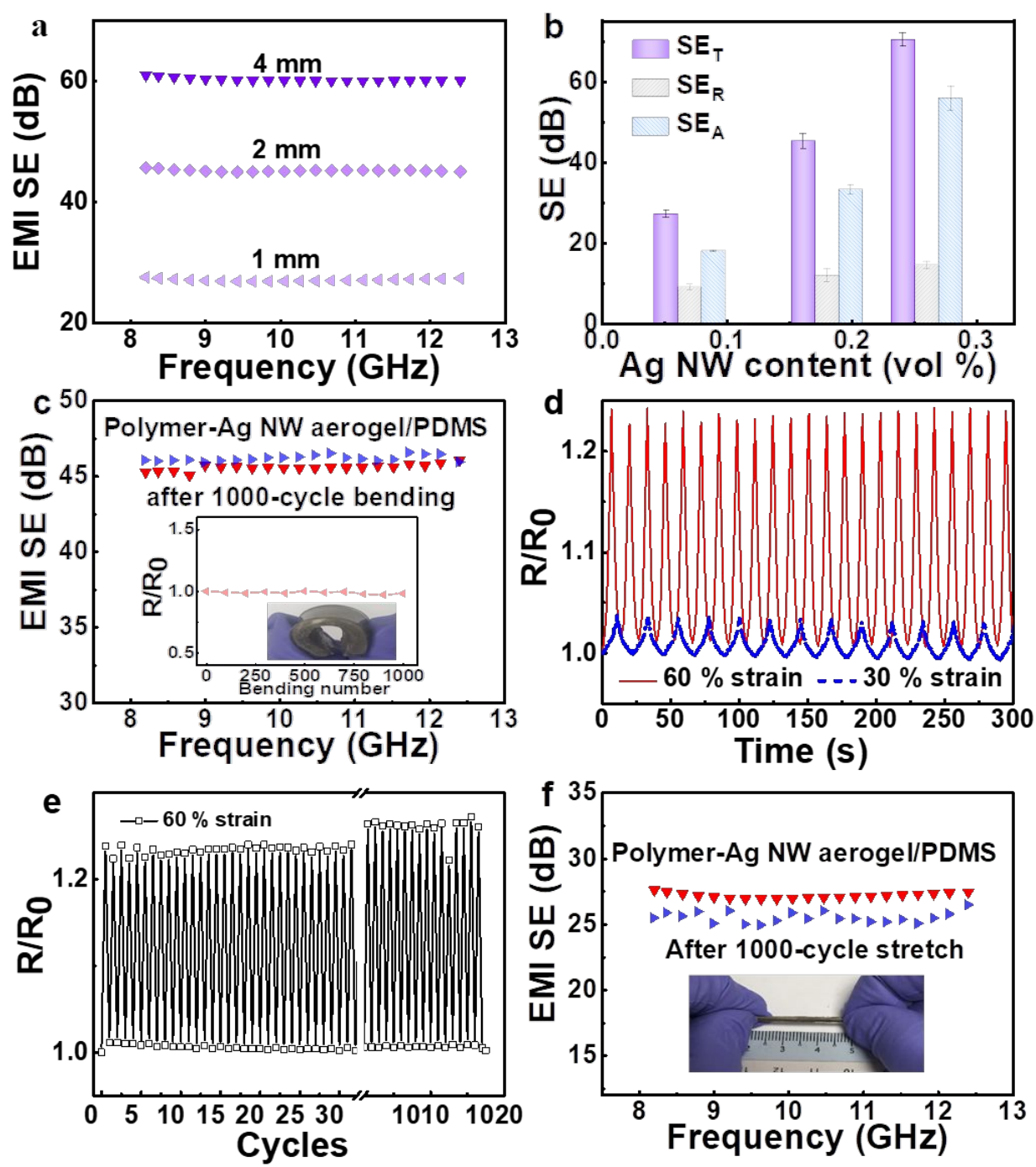

Figure 4. Properties of the reinforced AgNW aerogel/PDMS composites: (a) EMI SE at $10 \mathrm{GHz}$ of the samples with various thicknesses and (b) EMI shielding performance of the 2 mm-thick composites at various AgNW contents. (c) EMI SE and resistance ratio of the composites before and after bending (with a bending radius of around $10 \mathrm{~mm}$ ) process. Resistance ratio of the composites (d) at different cyclic tensile strains, and (e) in a long-time stretching/release cycling process at the strain of $60 \%$; (f) EMI SE of a 1 mm-thick sample before and after being stretched at a $60 \%$ strain for more than 1000 cycles. 


\section{Table of Contents / Graphic Abstract}
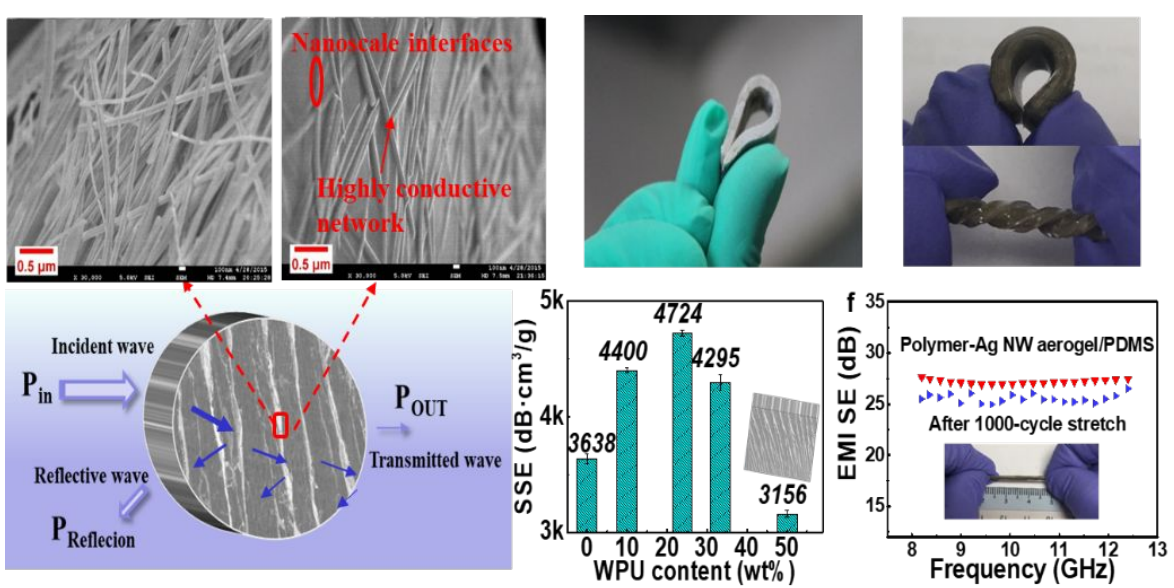